\title{
RELAÇÃO ENTRE SATISFAÇÃO E INSATISFAÇÃO COM A IMAGEM CORPORAL E INDICADORES ANTROPOMÉTRICOS: ESTUDO REA- LIZADO COM ESTUDANTES DA ESCOLA SECUNDÁRIA DE ES- TARREJA, NO DISTRITO DE AVEIRO
}

\author{
Maria Claudia Pinheiro \\ Instituto Universitário da Maia, Maia, Portugal \\ Sebastião Votre \\ Universidade do Estado do Rio de Janeiro, Rio de Janeiro, Rio de Janeiro, Brasil
}

\begin{abstract}
Resumo
O objetivo do presente estudo foi analisar a relação entre imagem corporal e grau de satisfação e insatisfação com ela, tendo em consideração as variáveis morfológicas sexo e Índice de Massa Corporal. Foram entrevistados 12 adolescentes (seis rapazes e seis garotas), divididos em três categorias, de acordo com o seu IMC. Verificamos que alguns dos nossos entrevistados consideraram estar longe do modelo de corpo ideal, mas afirmaram gostar dos seus corpos por habituação ou conformismo. Três dos entrevistados com excesso de peso mostraramse insatisfeitos com o peso e afirmaram desejar ser mais magros.
\end{abstract}

Palavras-chave: Imagem corporal. Satisfação. Adolescentes.

\section{Introdução}

A imagem corporal tem vindo a ser conceptualizada como sendo o desenho mental do nosso próprio corpo. Isto é, o resultado das perceções conscientes e inconscientes, dos sentimentos e das atitudes que produzimos relativamente ao nosso corpo ao longo da nossa existência. Gardner (1996) define imagem corporal como sendo a figura mental que temos das medidas, dos contornos e da forma do nosso corpo e dos sentimentos respeitantes a essas características e às partes do corpo. Partindo desta definição, pode-se afirmar que este conceito tem duas componentes: uma componente perceptiva, relacionada com a representação mental do corpo e com o grau de precisão dessa representação, e uma componente atitudinal, relacionada com os sentimentos acerca do corpo ou de partes do corpo. Isto é, uma componente mais afectiva e subjectiva, que se relaciona com o grau de satisfação ou insatisfação com o corpo ou partes do corpo (GARDNER, 1996; SANTOS et al., 2009).

Nas sociedades atuais, a indústria cultural e os meios de comunicação social são grandes responsáveis pela criação de desejos e pelo reforço de imagens padronizadas de corpo (GRAUP et al., 2008). Isto é, existe uma forte divulgação de que corpos magros para as mulheres e musculosos para os homens são sinónimo de sucesso, felicidade, alegria, bem-estar pessoal e aceitação social (DAMASCENO et al., 2005). Os indivíduos, ao serem expostos a estas imagens padronizadas de corpos, comparam e avaliam os seus corpos em relação a essas imagens e procuram atingir os ideais de beleza divulgados. É neste contexto que podem surgir 
conflitos entre o ideal de beleza prescrito pela sociedade e o somatótipo dos indivíduos, aumentando a predisposição para a insatisfação corporal. A insatisfação com a imagem corporal é uma das componentes da imagem corporal e pode ser definida como a "avaliação negativa do próprio corpo" (ADAMI et al., 2008, p. 143). Esta insatisfação está relacionada com as atitudes e avaliações do próprio corpo (GRABE; HYDE, 2006). Essa insatisfação, ainda que ocorrendo em diferentes idades, tende a ser preponderante durante a adolescência (ADAMI et al., 2008; BRANCO; HILÁRIO; CINTRA, 2006).

A adolescência é geralmente considerada um período difícil no que respeita à insatisfação corporal, pois é neste período que ocorrem alterações ao nível do desenvolvimento humano, assim como transformações nas dimensões corporais. Estas mudanças que ocorrem ao nível da forma e do esquema corporal podem interferir diretamente na construção da imagem corporal, levando vários jovens a desenvolverem sentimentos de insatisfação com o seu corpo ou partes do corpo, mesmo entre aqueles que se encontram dentro dos parâmetros considerados adequados para o seu grupo (BEARMAN et al., 2006; PINHEIRO; GIUGLIANI, 2006).

Durante o período da adolescência, as garotas tendem a demonstrar uma grande preocupação com a aparência. Nesta fase, elas procuram perder peso e adquirir uma forma corporal mais magra, na tentativa de se adequarem ao ideal de beleza padronizado, o qual se encontra fortemente associado à ideia de magreza (SMOLAK; LEVINE; THOMPSON, 2001). Nos rapazes, como o ideal de beleza tende a valorizar a musculatura, há aqueles que procuram aumentar o seu peso. No entanto, também há os que procuram diminuir o peso ou a forma corporal.

Neste contexto, o objetivo do presente estudo consistiu em analisar a perceção da imagem corporal e o grau de satisfação/insatisfação corporal em adolescentes de ambos os sexos da Escola Secundária de Estarreja, no distrito de Aveiro, e verificar possíveis relações com dois indicadores antropométricos (sexo e Índice de Massa Corporal - IMC).

\section{Métodos}

A investigação empregue neste estudo foi de índole qualitativa. Este tipo de investigação tem como objeto de estudo as intenções e situações. Por outras palavras, "procura-se investigar ideias, descobrir significados nas ações individuais e nas interações sociais a partir da perspetiva dos atores intervenientes no processo" (RESENDE et al., 2013, p. 522). A investigação qualitativa baseia-se no método indutivo, uma vez que o investigador tem como intuito desvendar o propósito da ação.

\section{Participantes}

Numa primeira fase, optámos por escolher trabalhar com os 37 alunos da Escola Secundária de Estarreja, do distrito de Aveiro, que tinham 16 anos de idade. Escolhemos esta fase da adolescência por se tratar de jovens capazes de transmitir as suas ideias com clareza e capacidade de entendimento e por estarem numa fase da vida na qual se assiste a uma série de modificações que se traduzem em alterações morfológicas, funcionais e comportamentais, com um grande significado psicológico e social. Uma segunda seleção foi efetuada com base no cálculo do Índice de Massa Corporal (IMC), o qual relaciona o peso corporal com o quadrado da altura. Após ter sido calculado o IMC de todos os 37 jovens de 16 anos, e com base na classificação da obesidade segundo o IMC (World Health Organization, 2015), os indivíduos foram reunidos em três grupos: com peso abaixo da média (Peso baixo (pb) - IMC C 18,5), com peso ideal (Peso normal (pn) - IMC entre 18,5 e 24,9) e com peso acima da média (Peso em excesso (pe) - IMC $\geq 25$ ). Após estas medições, foram selecionados aleatoriamente 
12 alunos (dois rapazes e duas garotas com baixo peso, dois rapazes e duas garotas com peso normal e dois rapazes e duas garotas com excesso de peso).

Tabela 1 - Grupo de estudo

\begin{tabular}{llll}
\hline & \multicolumn{3}{c}{ Entrevistados } \\
\hline Sujeitos & Gênero & IMC & \multicolumn{1}{c}{ Classificação } \\
\hline E1 & $\sigma^{\prime}$ & 40,32 & Peso em excesso (pe) \\
E2 & $\sigma^{\prime}$ & 24,82 & Peso normal (pn) \\
E3 & $\sigma^{\prime}$ & 19,23 & Peso normal (pn) \\
E4 & $\sigma^{\prime}$ & 29,03 & Peso em excesso (pe) \\
E5 & $\sigma^{\prime}$ & 17,9 & Peso baixo (pb) \\
E6 & $\wp$ & 17,6 & Peso baixo (pb) \\
E7 & $\wp$ & 18,23 & Peso baixo (pb) \\
E8 & $\wp$ & 30,44 & Peso em excesso (pe) \\
E9 & $\wp$ & 18,6 & Peso normal (pn) \\
E10 & $\wp$ & 28,6 & Peso em excesso (pe) \\
E11 & $\wp$ & 24,5 & Peso normal (pn) \\
E12 & $\bigcirc^{\prime}$ & 18,14 & Peso baixo (pb) \\
\hline
\end{tabular}

Fonte: Elaborado pelos autores, 2016

\section{Recolha da informação}

A presente investigação que visa ao estudo das perceções utiliza a abordagem direta dos sujeitos nos seus próprios contextos de interação - no gênero discursivo entrevista. A entrevista permite recolher diretamente informações relativas ao objeto de estudo e possibilita a obtenção de um elevado grau de profundidade dos elementos em análise, nomeadamente sobre comportamentos (GIL, 2008), admitindo maior flexibilidade na sequência das questões, o que permite que a recolha de informação seja efetuada respeitando o quadro de referência do entrevistado (QUIVY; CAMPENHOUDT, 2008). Para Gil (2008), a entrevista consiste num diálogo que se estabelece entre uma parte que procura obter informações e uma parte que se apresenta como fonte de informação.

Para analisar a perceção da imagem corporal e o grau de satisfação e insatisfação corporal, recorreu-se a uma entrevista semiestruturada. Este tipo de entrevista, de acordo com Flick (2005), exige um envolvimento bastante estreito entre investigadores e entrevistados. Nessa entrevista, o investigador assume a função de orientador do processo, cabendo-lhe a decisão da sequência e da redação das questões, bem como o encaminhamento da entrevista para os temas considerados relevantes (QUIVY; CAMPENHOUDT, 2008). O roteiro de questões foi elaborado com base na fundamentação conceitual que sustenta a problemática, bem como nos objetivos do presente estudo. O roteiro foi previamente validado por método de peritagem, por meio de dois especialistas em investigação na área da Educação Física.

A anteceder a realização das entrevistas, foi solicitada autorização à direção da escola para a realização da investigação. Posteriormente, solicitou-se aos encarregados de educação autorização para a realização e a gravação de uma entrevista com os seus educandos. Estes foram informados dos objetivos do trabalho, bem como lhes foi garantido que a sua dignidade e o sigilo seriam totalmente respeitados. Após a obtenção do consentimento livre e esclarecido, procedeu-se à realização das entrevistas. 


\section{Análise da informação}

Os dados recolhidos, depois de transcritos verbatim, foram submetidos à técnica de análise de conteúdo. Esta técnica pressupõe a codificação da informação como sendo uma fase decisiva da investigação qualitativa. A codificação é, de acordo com Neuman (2003), a organização da informação em bruto em categorias conceptuais para se poderem criar temas ou conceitos que facilitem a análise da informação. De acordo com Bardin (2008, p. 39), a categorização consiste em "uma operação de classificação de elementos constitutivos de um conjunto por diferenciação". Este processo de categorização teve por base o roteiro da entrevista. Deste modo, as categorias de análise foram definidas a priori assentando na fundamentação teórica recolhida na fase inicial da investigação.

As cinco categorias de análise foram as seguintes: perceção da imagem corporal, perceção do seu corpo através do olhar do outro, grau de satisfação/insatisfação com o seu corpo, modelo de corpo "ideal", aproximação do seu corpo em relação ao modelo de corpo "ideal".

\section{Resultados e discussão}

\section{A - Perceção da imagem corporal}

Conforme mencionado anteriormente, o conceito de imagem corporal tem duas componentes: uma componente atitudinal, de natureza mais subjetiva (que inclui atitudes acerca do tamanho, do peso, das partes corporais e da aparência em geral), e uma componente preceptiva relacionada com a representação mental do corpo (perceção do tamanho do corpo e outros detalhes). Foi sobre esta componente percetiva que se centrou a questão colocada aos nossos entrevistados. Ou seja, procurámos saber qual a perceção dos alunos em relação ao seu tamanho corporal. Assim, verificamos que os entrevistados de ambos os sexos classificados com excesso de peso se percecionaram como sendo "gordos". Tendência semelhante foi encontrada com as alunas classificadas com baixo peso, as quais se percecionaram como sendo magras.

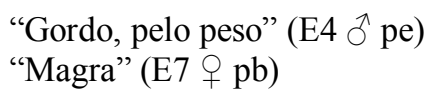

Veloso, Matos e Veloso (2009) verificaram que as moças têm geralmente uma perceção do seu corpo que frequentemente não corresponde à realidade. Têm a propensão para se verem mais gordas do que na realidade são, ao contrário dos rapazes, que têm tendência para se verem mais magros. O nosso estudo apresenta, porém, resultados contrários. Os entrevistados classificados com baixo peso consideram ter o peso ideal, não se percecionando como magros, mas sim mais gordos do que na realidade o IMC indica.

\footnotetext{
"Acho que estou dentro das medidas certas" (E12 $\hat{\rho} \mathrm{pb})$

"Sinto-me no peso ideal" (E5 $\widehat{\jmath} \mathrm{pb})$
}

Já no grupo classificado como tendo peso normal, encontrámos opiniões diferenciadas, como se pode verificar através das falas de dois dos nossos entrevistados, sendo um do sexo feminino e outro do sexo masculino.

“Gorda, só um pouco" (E11, + pn)

"Um bocadinho gordo porque já não faço exercício como antigamente” (E2, đ̂ pn) 
Estas duas falas revelam que a imagem mental que temos do nosso corpo nem sempre corresponde ao que na verdade somos.

\section{B - Perceção do seu corpo através do olhar do outro}

Segundo Turner (1992), a imagem corporal é "um corpo para os outros", a perceção que cada um tem do seu corpo resulta do uso como fonte primária e como depósito de símbolos para relações sociais. Somos o que os outros veem, o ver e o ser visto tornaram-se comportamentos individuais, ou seja, o ser humano transformou-se no "olhar" (LE BRETON, 1992), deixando o corpo de ser dimensão privada para se tornar pública. Quando se perguntou aos alunos o que achavam da opinião dos seus pares relativamente ao seu corpo (o corpo que é visto), as respostas levam-nos a concluir que o fosso existente entre o corpo que se vê e o corpo que se percepciona não é muito grande.

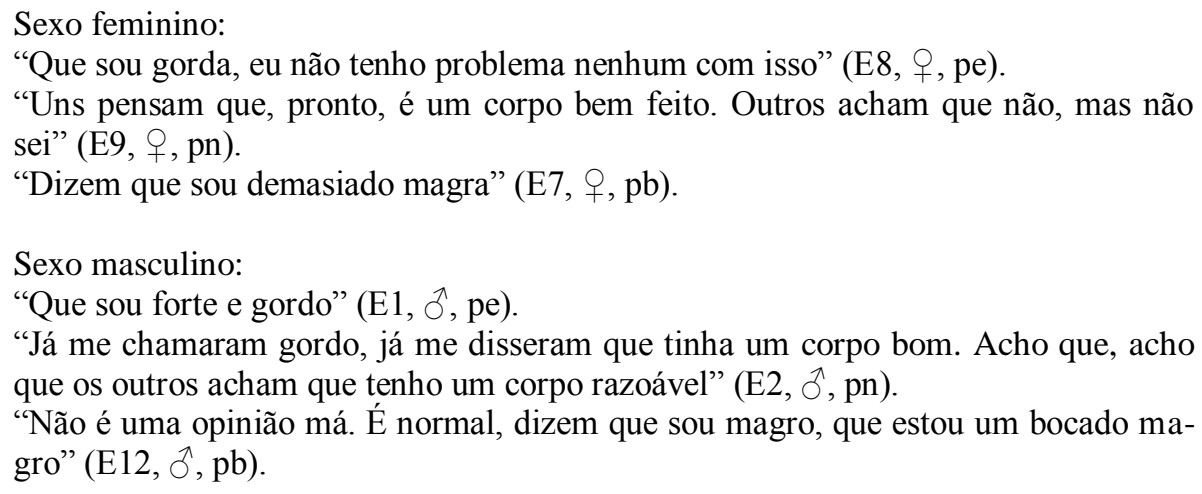

Depreendemos que a opinião dos pares é importante para os adolescentes, uma vez que os entrevistados têm a noção da opinião dos outros relativamente ao seu corpo. Por norma, os adolescentes emitem juízos de valor sobre os atributos físicos, fazem apreciações estéticas sobre o corpo e criticam o outro. Estas atitudes fazem com que a necessidade de afirmação pessoal e o apreço por parte dos amigos sejam valorizados, já que a relação que temos com o corpo não é meramente uma questão estética, é também a forma de mostrar a ligação que se tem consigo mesmo (RIBEIRO, 2005).

Os discursos apresentados sustentam a opinião de Gomes, Silva e Queirós (2000), ao afirmarem que o nosso corpo é o nosso cartão de visita, é a nossa apresentação, a nossa primeira impressão. Ribeiro (2005) refere que, quando olhamos para um desconhecido, é através da sua aparência física que retiramos as primeiras impressões, podendo ou não despertar em nós a vontade de conhecê-lo. Se tal acontecer, é provável que a importância que damos à sua aparência física diminua e dê lugar à elevação dos valores morais, sentimentos e atitudes.

\section{C - Grau de satisfação/insatisfação com o seu corpo}

Quando questionamos os nossos entrevistados acerca do que pensam do seu próprio corpo, verificamos que, independentemente do IMC, 9 dos 12 inquiridos mostraram-se conformados com os seus corpos:

\footnotetext{
"Gosto. Já me habituei a ele" (E6, † pb)

"Já gostei menos. Agora gosto do meu corpo, sim gosto" (E8, $ๆ$ pe)

“Sim. Já estou habituado a ele" (E12, ô pb)
}

Contudo, dois inquiridos referiram não gostar (um com peso normal e outro com excesso de peso) e um terceiro (com excesso de peso) mencionou gostar algumas vezes: 


\begin{abstract}
"Não. Acho que estou um pouquinho gorda" (E11,, pn).
"Não gosto. Pelas razões que já disse (ancas, excesso de peso, pele mais branca e peito mais pequeno), já falei da gordura e da pele" (E10,, pe).

"Às vezes sim, outras vezes não. Porque em certas alturas ele é útil, mas noutras alturas ele não é. Ele é útil para alguns tipos de trabalhos que eu faço em casa, mas para outros trabalhos não é útil porque eu tenho dificuldades em baixar-me para fazer certas coisas" (E1, ठ̂ pe).
\end{abstract}

Verificamos que os resultados encontrados vão ao encontro do já evidenciado em outros estudos (CASH; BROWN, 1989; FRANZOI; KESSENICH; SUGRUE, 1989; RICHARDS et al., 1990), os quais referem, tal como observado no nosso trabalho, que o sexo masculino, independentemente do seu IMC e de uma forma geral, está mais satisfeito com a sua imagem corporal do que o sexo feminino. Das duas adolescentes com peso normal, uma encontra-se satisfeita com o seu peso e a outra encontra-se algo insatisfeita com o seu peso, o que se encontra na mesma linha de ideias de outros estudos (BRANCO et al., 2006; SANTOS et al., 2009), os quais referem que adolescentes do sexo feminino, mesmo com peso normal, tendem a apresentar insatisfação com o peso que têm.

\title{
D - Modelo de corpo "ideal"
}

É com o nosso corpo que percebemos o mundo e a nós próprios. É com esse mesmo corpo que nos expomos a críticas sociais e é do corpo dos outros que falamos quando o observamos. Falar de um corpo saudável, bonito, alto, magro, tonificado e jovem faz parte do modelo de corpo "ideal" socialmente aceite (CASH; BROWN, 1989; FRANZOI; KESSENICH; SUGRUE, 1989; LOVISOLO, 1997; RICHARDS et al., 1990). Quando se perguntou aos alunos se consideram que existe um modelo de corpo "ideal", as respostas dividiram-se:

\footnotetext{
"Ideal? Assim perfeito? Acho que não" (E3, ơ pn).

"Sim há um corpo ideal" (E5, ô pb).

"Eu não acho que exista um corpo ideal" (E7,, pb).

"Houve e há. Ou seja, antes eram as mais gorduchinhas, hoje em dia são as mais elegantes, as mais altas" (E11, + pn).
}

Quando pedimos aos adolescentes para descreverem o corpo "ideal" para um homem e para uma mulher, independentemente do seu sexo, verificamos que, para a mulher, o corpo ideal é o determinado pela sociedade atual (magro, alto, bonito e jovem), enquanto, para o homem, se aponta para um corpo musculoso, tonificado e alto. Os nossos entrevistados sugerem, assim como Veloso, Matos e Veloso (2009), que o corpo ideal para uma garota serve como meio de atrair os outros (sendo alta, magra, bonita), contrariamente ao corpo ideal preconizado para os rapazes, a quem serve como modo eficaz de atuar no meio, um instrumento onde os músculos são necessários.

\section{E - Aproximação do seu corpo em relação ao modelo de corpo "ideal"}

Quando questionados acerca da possível proximidade do seu corpo em relação ao modelo de corpo "ideal", os alunos deram respostas praticamente unânimes (sexo e IMC), ao considerar que não têm esse corpo, conforme se pode observar através do seu próprio discurso:

Sexo feminino: 


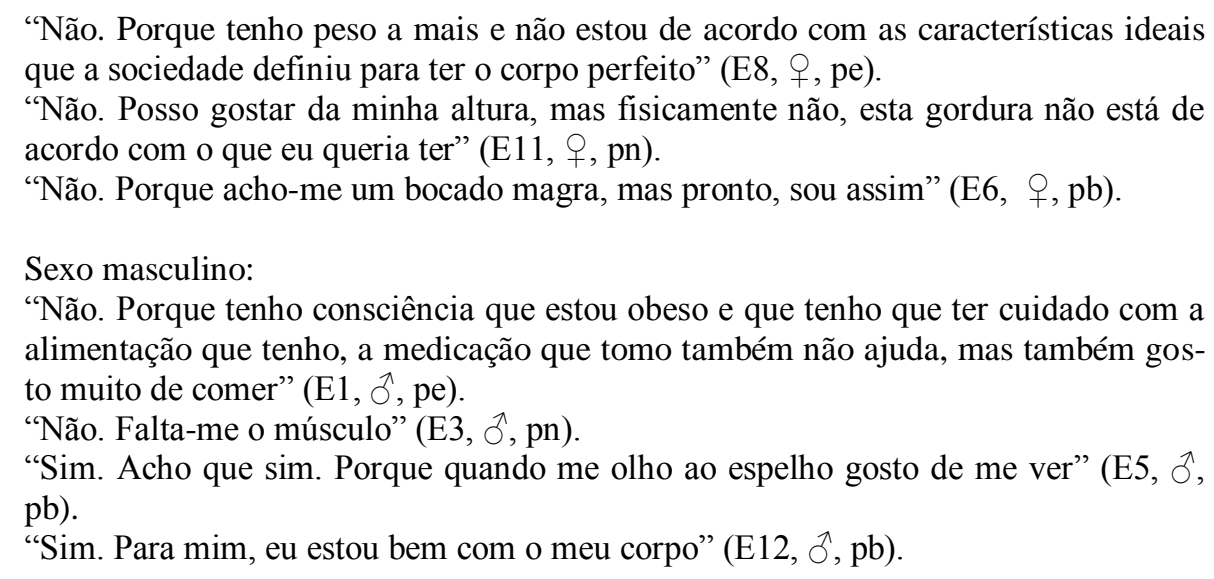

No que concerne ao IMC, podemos verificar que tanto obesidade como baixo peso estão presentes nos alunos e nas alunas, respetivamente classificados com excesso de peso e com baixo peso. Os rapazes classificados com baixo peso foram os únicos a afirmar que já têm um corpo ideal, este corpo agrada-lhes e a habituação a ele acaba por fazê-los acreditar que é um corpo ideal.

Dado vários dos nossos entrevistados terem afirmado não ter um corpo ideal e se encontrar longe do modelo de corpo "ideal", consideramos ser pertinente inferir o que estes jovens gostariam de modificar nos seus corpos e o que estariam dispostos a fazer para se aproximar do modelo de corpo tão frequentemente divulgado.

Verificamos que as adolescentes ambicionam ser magras e eles mais musculosos. As garotas pretendem atingir um peso abaixo daquele que têm, enquanto os rapazes preferem aumentar a massa muscular.



No que respeita ao corpo "ideal", verificamos que os rapazes classificados com peso em excesso ambicionam ser mais magros, não referindo à massa muscular como pretensão. $\mathrm{O}$ mesmo acontece com as garotas classificadas com peso baixo, as quais ambicionam ser mais fortes/gordas e mais altas.

"Ser mais magro" (E1, ô, pe).

"Um pouco mais magro" (E4, §̂, pe).

“Apenas ser um bocadinho mais forte, talvez um bocadinho mais alta" (E7, $q, \mathrm{pb})$.

"Talvez gostava de ser um bocadinho mais gorda e um pouco mais alta" (E6,, , pb).

Quando questionados sobre o que estariam dispostos a fazer para tentar alcançar o dito modelo de corpo "ideal", três dos entrevistados classificados com peso normal e três dos adolescentes de ambos os sexos classificados com peso em excesso afirmam que o exercício físico, uma alimentação saudável e as dietas seriam a melhor opção para alcançar o referido corpo.

\footnotetext{
"Treinar mais, esforçar-me mais, trabalhar com o corpo" (E2, §ે, pn).

"Praticar exercício físico, comer coisas saudáveis" (E9, ㅇ pn).

“Alimentação, exercício físico" (E11, q pn).

"Fazer dieta" (E1, ô, pe).

"Uma dieta e exercício físico" (E4, ô, pe).

"Comer menos, fazer mais exercícios" (E10, q pe).
} 
Contudo, dois dos entrevistados (um com peso normal e outro com excesso de peso) afirmam não pretender alcançar o dito corpo "ideal", por se sentirem bem com o corpo que têm ou por considerarem que tal significa uma conformidade dos indivíduos a padrões impostos por outros.

"Não trabalho para isso" (E5, ô, pb).

"Não. Porque isso é ir de encontro ao que as pessoas pensam, a sociedade ... assim não vão a lado nenhum ... as pessoas são fúteis" (E8, + pe).

\section{Conclusões}

Cada sociedade difunde um tipo de modelo de corpo "ideal", o qual tende a ser incorporado pelos adolescentes. Na busca de interpretação para os processos de incorporação, elegemos como categorias analíticas a perceção da imagem corporal, a perceção do seu corpo através do olhar do outro, o grau de satisfação/insatisfação com o seu corpo, o modelo de corpo "ideal" e a aproximação do seu corpo em relação ao modelo de corpo "ideal". Assim, independentemente da classificação de IMC, compreendemos através dos discursos dos nossos alunos que, para a maioria dos entrevistados, o corpo serve de embrulho; o seu significado é mesmo o de "cartão de visitas", aquilo que se apresenta ao mundo.

Para estes adolescentes, o modelo de corpo "ideal" é o preconizado pelos organismos sociais fazedores de opinião. O corpo "ideal" para uma mulher é magro, alto e jovem e, para o homem, aponta-se para um corpo musculado, tonificado e alto. É por descrever dessa forma o corpo "ideal" que o nosso grupo de estudo considera que não o tem, à exceção dos rapazes considerados com baixo peso.

Uma das garotas e os dois rapazes classificados com excesso de peso mostram-se insatisfeitos com o seu corpo, têm consciência da obesidade e desejariam ser mais magros, estando dispostos a fazer dietas controladas e exercício físico.

Os resultados encontrados parecem indicar que os rapazes classificados com peso normal consideram que não detêm um corpo "ideal", pois sentem que não têm os músculos bem definidos. Os rapazes apontam o exercício físico como o meio para alcançar o corpo "ideal", porém, afirmam não pretender realizar qualquer alteração dos seus corpos. Já as garotas classificadas com peso normal apontam para as questões estéticas, afirmando não ter o corpo "ideal", pois consideram que acumularam gorduras em certas partes do corpo e que, como tal, alterariam certos aspetos corporais através do exercício físico, da alimentação saudável e de operações estéticas. As garotas com baixo peso reconhecem que são magras. Desejariam ser um pouco mais fortes e altas para se aproximarem do modelo de corpo ideal. Os classificados com baixo peso de gênero masculino consideram que o seu corpo é o ideal, não por ser um corpo musculado, mas por se identificarem com ele.

Podemos afirmar que alguns dos nossos entrevistados consideram estar longe do modelo de corpo "ideal", mas afirmaram gostar dos seus corpos por habituação ou conformismo. Percecionam o seu corpo em conformidade com o seu peso e creem que os outros também os veem do mesmo modo. Por exemplo, quem tem excesso de peso perceciona-se como sendo "gordo" e afirma que os outros detêm essa opinião sobre ele.

As palavras dos entrevistados evidenciam a manifesta crise de identidade que coincide com a puberdade e estimula a insatisfação com a imagem corporal. Enquanto alguns dos adolescentes ambicionam um corpo ideal, outros mostram-se satisfeitos com o corpo que têm.

Os resultados, apesar de, aparentemente, poderem parecer muito módicos, atendendo ao número de entrevistados, dão-nos uma primeira informação sobre a perceção da imagem corporal e os níveis de satisfação e insatisfação com a imagem corporal de adolescentes de ambos os sexos. 
Perante estes resultados, torna-se importante que profissionais na área da saúde e da educação incentivem os jovens a conhecerem melhor os seus corpos e a entenderem as relações que os seus corpos têm com a saúde e com a qualidade de vida. É também fundamental que estes jovens aprendam a refletir sobre os padrões de beleza corporal em voga e compreendam que, por vezes, a busca desses padrões de beleza pode ser prejudicial à saúde. Neste contexto, os profissionais de educação e de educação física podem ser importantes agentes, na medida em que poderão desenvolver programas de intervenção direcionados para esta faixa etária que, para além de terem como objetivo o aumento dos níveis de atividade física, contribuam simultaneamente para uma melhoria dos níveis de satisfação com o seu próprio corpo. Para além destes programas, poderão ser criados nas escolas, e com a ajuda dos diferentes profissionais da educação, contextos favoráveis à discussão e à reflexão sobre a imagem corporal e o papel que a educação física pode ter no desenvolvimento da perceção que cada um tem do seu corpo.

\title{
RELATIONSHIP BETWEEN BODY IMAGE SATISFACTION, DISSATISFACTION AND ANTHROPOMETRIC INDICATORS: STUDY CONDUCTED WITH STU- DENTS FROM THE ESTARREJA SECONDARY SCHOOL IN THE DISTRICT OF AVEIRO
}

\begin{abstract}
The aim of this study was to analyze the relation between body image and levels of body satisfaction and dissatisfaction taking into account sex and body mass index. 12 children ( 6 boys and 6 girls) divided into 3 categories according to their BMI were interviewed. We found that some of our respondents considered to be away from the model of the ideal body but claimed to like their bodies for habituation or conformism. 3 of our interviewees with excessive weight were dissatisfied with their body weight and would like to be thinner.
\end{abstract}

Keywords: Body image. Satisfaction. Adolescents

\section{RELACIÓN ENTRE LA SATISFACCIÓN Y LA INSATISFACCIÓN CON LA IMA- GEN CORPORAL, Y EL ESTUDIO DE INDICADORES ANTROPOMÉTRICOS DE LOS ESTUDIANTES DE LA ESCUELA SECUNDARIA ESTARREJA EN EL DIS- TRITO DE AVEIRO}

\begin{abstract}
Resumen
El objetivo de este estudio fue analizar la relación de la imagen corporal y la satisfacción y la insatisfacción corporal considerando el índice de masa corporal y los sexos. Entrevistamos a 12 niños (6 niños y 6 niñas) divididos en 3 categorías según su índice de masa corporal. Encontramos que algunos de nuestros encuestados consideran estar lejos del modelo del cuerpo ideal, pero afirmaron gustar de sus cuerpos por habituación o conformismo. 3 de nuestros entrevistados con exceso de peso no estaban satisfechos con su peso corporal y les gustaría ser más delgados.
\end{abstract}

Palabras clave: Imagen corporal. La satisfacción corporal. Adolescentes

\section{Referências}


ADAMI, F. et al. Insatisfação corporal e atividade física em adolescentes da região continental de Florianópolis. Psicologia: Teoria e Pesquisa, v. 24, n. 2, p. 143-149, 2008.

BARDIN, L. Análise de conteúdo. Lisboa: Edições 70, 2008.

BEARMAN, S. K. et al. The skinny on body dissatisfaction: a longitudinal study of adolescent girls and boys. Journal of Youth and Adolescence, New York, v. 35, n. 2, p. 229-241, 2006.

BRANCO, L.; HILÁRIO, M. O.; CINTRA, I. Percepção e satisfação corporal em adolescentes e a relação com seu estado nutricional. Revista de Psiquiatria Clínica, v. 33, n. 6, p. 292296, 2006.

CASH, T.; BROWN, T.. Gender and body images: stereotypes and realities. Sex Roles, New York, v. 21, n. 5-6, p. 361-373, 1989.

DAMASCENO, V. et al. Tipo físico ideal e satisfação com a imagem corporal de praticantes de caminhada. Revista Brasileira de Medicina no Esporte, v. 11, n. 3, p. 181-186, 2005.

FLICK, U. Métodos qualitativos na investigação científica. Lisboa: Monitor, 2005.

FRANZOI, S.; KESSENICH, J.; SUGRUE, P. Gender differences in the experience of body awareness: an experimental sampling study. Sex Roles, New York, v. 21, n. 7-8, p. 449-541, 1989.

GARDNER, R. Methodological issues in assessment of the perceptual component of body image disturbance. British Journal of Psychology, n. 87, p. 327-337, 1996.

GIL, A. Métodos e técnicas de pesquisa social. São Paulo; Atlas, 2008.

GOMES, P.; SILVA, P.; QUEIRÓS, P. Equidade na educação, educação física e desporto na escola. Queijas: Associação Portuguesa A Mulher e o Desporto, 2000.

GRABE, S.; HYDE, J. Ethnicity and body dissatisfaction among women in the United States: a meta-analysis. Psychology Bulletin, Washington, v. 132, n. 4, p. 622-640, 2006.

GRAUP, S. et al. Associação entre a percepção da imagem corporal e indicadores antropométricos de escolares. Revista Brasileira de Educação Física e Esporte, v. 22, n. 2, p. 129-138, 2008.

LE BRETON, D. Anthropologie du corps et modernité. Paris: Presses Universitaires de France, 1992.

LOVISOLO, H.. Estética, exporte e educação física: ensaios. Rio de Janeiro: Sprint Editora, 1997.

NEUMAN, W.. Social research methods: qualitative and quantitative approaches. Boston: Pearson Education, 2003. 
PINHEIRO, A.P.; GIUGLIANI, E. R. Quem são as crianças que se sentem gordas apesar de terem peso adequado? Jornal de Pediatria, v. 82, n. 3, p. 232-235, 2006.

QUIVY, R.; CAMPENHOUDT, L. Manual de investigação em ciências sociais. Lisboa: Gradiva Publicações, 2008.

RESENDE, R. et al. A percepção dos professores cooperantes sobre os conhecimentos e as competências dos estudantes. Revista de Educação Física/UEM, v. 24, n. 4, p. 519-533, 2013.

RIBEIRO, A. O corpo que somos. Aparência, sensualidade, comunicação. Cruz Quebrada: Casa das Letras, 2005.

RICHARDS, M. et al. Relation of weight to body image in pubertal girls and boys from two communities. Developmental Psychology, Washington, v. 26, n. 2, p. 313-321, 1990.

SANTOS, M. et al. Imagem corporal e níveis de insatisfação em adolescentes na pósmenarca. Revista de Educação Física/UEM, v. 20, n. 3, p. 333-341, 2009.

SMOLAK, L.; LEVINE, M.; THOMPSON, K. The use of the sociocultural attitudes towards appearance questionnaire with middle school boys and girls. International Journal of Eating Disorders, Middleton, v. 29, n. 2, p. 216-223, 2001.

TURNER, B. Regulating bodies: essays in medical sociology. London: Routledge, 1992.

VELOSO, S.; MATOS, M.; VELOSO, N. O crescimento e o corpo. In: MATOS, M.; SAMPAIO, D. (Orgs.) Jovens com saúde - diálogo com uma geração. Lisboa: Textos Editores, Lda, 2009.

WHO. World Health Organization. BMI classification. 2015. Disponível em: <http://apps.who.int/bmi/index.jsp?introPage=intro 3.html >. Acesso em: 2 maio 2015.

Recebido em: 01/09/2016

Revisado em: 11/11/2017

Aprovado em: 10/12/2017

Endereço para correspondência:

pinclaudia@gmail.com

Maria Claudia Pinheiro

Av. Carlos de Oliveira Campos, s/n

Castêlo da Maia

4475-690 - Maia - Portugal 